\title{
In silico assessment of the inhibitory effect of four flavonoids (chrysin, naringin, quercetin, kaempferol) on tyrosinase activity using the MD simulation approach
}

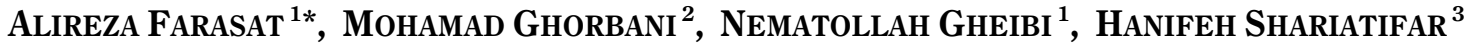 \\ ${ }^{1}$ Cellular and Molecular Research Center, Research Institute for Prevention of Non-Communicable Diseases, \\ Qazvin University of Medical Sciences, Qazvin, Iran \\ ${ }^{2}$ Department of Nanobiotechnology/Biophysics, Faculty of Biological Science, Tarbiat Modares University, Tehran, Iran \\ ${ }^{3}$ Young Researchers and Elite Club, Tehran Medical Sciences, Islamic Azad University, Tehran, Iran
}

\begin{abstract}
Tyrosinase is a tetrameric enzyme that plays an important role in pigment production. Overproduction of melanin, which may lead to several skin disorders, is a result of tyrosinase activity. Hence, tyrosinase inhibitors are of key importance in the treatment of these disorders. In the present study, four flavonoid inhibitors, namely chrysin, naringin, quercetin, and kaempferol, were evaluated physiochemically, and the inhibitory effects of these compounds on tyrosinase activity were evaluated using the molecular dynamics (MD) simulation method. To create the best conformation of the enzyme-substrate/inhibitor, the docking process for enzyme-substrate, i.e., enzymechrysin, enzyme-quercetin, enzyme-naringin, and enzyme-kaempferol, was performed. The complexes with the best binding energies were selected as the models for the MD simulation process. Furthermore, the structural (RMSD, Rg, RMSF, and Distance) and the thermodynamics properties of the complexes were evaluated. Additionally, the PMF was conducted to calculate the binding free energies. The results showed that chrysin, quercetin and the substrate were at similar distances to the amino acids of the active site, but naringin and kaempferol were closer to the active site of the enzyme than the substrate. Moreover, the analysis of the binding energy revealed that the substrates, chrysin, kaempferol, quercetin, and naringin bound to the enzyme with binding energies of $-7.8,-3.1,-7.1,-3.9$, and $-8.4 \mathrm{kcal} / \mathrm{mol}$, respectively, which confirms that naringin has the highest inhibitory effect on tyrosinase among other inhibitors, which makes it an appropriate candidate as a whitening agent in skin disorders.
\end{abstract}

Key words: tyrosinase, chrysin, quercetin, kaempferol, naringin, MD simulation

$\begin{array}{lll}\text { Abbreviations } & \\ \text { COM } & \text { - center of mass } & \text { RMSF } \\ \text { L-DOPA } & \text { - L-3,4-dihydroxyphenylalanine } & \text { RMSD } \\ \text { MD } & \text { - molecular dynamics } & \text { Rg } \\ \text { PDB } & \text { - protein data bank } & \text { TIP3P } \\ \text { PMF } & \text { - potential of mean force } & \text { WHAM }\end{array}$

\section{Introduction}

Tyrosinase is a tetrameric $\left(\mathrm{H}_{2} \mathrm{~L}_{2}\right)$ copper-containing enzyme with significant catalytic functions in pigment production. The enzyme has molecular weight of $120 \mathrm{kDa}$ with two heavy $(\mathrm{H})$ and two light $(\mathrm{L})$ subunits of 43 and $14 \mathrm{kDa}$, respectively. Subunit H, contrary to subunit L,
- root mean square fluctuation

- root mean square deviation

- radius of gyration

- transferable intermolecular potential with 3 points

- weighted histogram analysis method

\footnotetext{
* Corresponding author: Cellular and Molecular Research Center, Qazvin University of Medical Sciences, Qazvin, Iran; e-mail: Farasat8@gmail.com
} 
figurations of the enzyme have been established during the melanin pigment production: oxy-tyrosinase, mettyrosinase, and deoxy-tyrosinase. The oxy form contains two copper atoms and one oxygen atom as a bridge between them. The met form is a resting enzymatic form, in which two cupric ions are bridged with one or two small ligands such as water molecules or hydroxide ions. The deoxy type is similar to the met form but lacks the hydroxide bridges (Abdelrasool and Said, 2017; Chang, 2009; Choi et al., 2017). Importantly, this enzyme catalyzes the hydroxylation reactions, leading to the formation of melanin through 3,4-dihydroxyphenylalanine (L-DOPA) and L-DOPA-quinone (Gheibi et al., 2016). Quinines can react with proteins and amino acids to form black or brown products (Sugumaran and Barek, 2016) or evolve chemically to form polyphenolic compounds or melanin (Gheibi et al., 2016; Ullah et al., 2016). Moreover, tyrosinase plays a key role in different processes such as melanin production in the skin, wound healing, agriculture, and cosmetic industry (Choi and Shin, 2016; Popoola et al., 2015; Taherkhani and Gheibi, 2014). The overactivity of the enzyme in the skin may lead to melanin overproduction and multiple skin disorders, including hyperpigmentation, depigmentation, and even melanoma (Choi and Shin, 2016; Popoola et al., 2015; Taherkhani and Gheibi, 2014). Among the various sources of tyrosinase, mushroom tyrosinase from Agaricus bisporus is a major and commercial source with high homology compared to human tyrosinase (Zolghadri et al., 2019). Because of these benefits, the structural, functional, and biochemical characteristics of mushroom tyrosinase have been studied extensively for screening tyrosinase inhibitors and melanogenic studies (Da Hae et al., 2019; Deri et al., 2016; Zolghadri et al., 2019). The melanin formation process can be influenced by multiple mechanisms, including direct inhibition of the enzyme or the inhibition of melanin movement from one cell to another (Taherkhani and Gheibi, 2014). Several studies have indicated that tyrosinase inhibitors are classified in four groups on the basis of their mechanism of action: 1) competitive inhibitors, 2) uncompetitive inhibitors, 3) competitive-uncompetitive inhibitors, and 4) noncompetitive inhibitors (Chang, 2009; Lin et al., 2017). Flavonoids are a major group of phenolic compounds with certain clinical characteristics such as antiallergic, anti-inflammatory, antifungal, antiviral, and antitumor properties, and they have recently gained more attention in cancer treatment (Harris et al., 2016; Horáková, 2011; Qiu et al., 2018). Four important flavonoid compounds, namely chrysin, quercetin, naringin, and kaempferol, have been proved to play a significant role in tyrosinase inhibition. Chrysin (5,7-dihydroxyflavone) is a natural flavonoid derived from many plants (Passiflora caerulea, Passiflora incarnata, and Oroxylum indicum) (Morissette et al., 2018) and possesses anti-melanogenesis effects (Zhu et al., 2016). Naringin (5,7,4-trihydroxyflavone) is another type of flavonoid with tyrosinase inhibition activity (Liu Smith and Meyskens, 2016). Quercetin (5,7,3,4-tetrahydroxyflavonol) also shows tyrosinase inhibitory effects through the inhibition of diphenolase activity (Harris et al., 2016). Kaempferol (5,7,4-trihydroxyflavonol) is another type of flavonoid inhibitor who antityrosinase effects have been proved frequently (da Silva et al., 2017; Promden et al., 2018; Solimine et al., 2016).

Today, studying the three-dimensional structures of proteins and protein-protein interactions is an important part of research on biology and drug design. Molecular dynamics (MD) simulation is an advanced method in evaluating macromolecular complexes such as proteins, ribosomes, and nucleosomes, and it could be applied in various reactions such as determination of drug molecule binding sites and their mechanisms, the mechanism of functional proteins, protein folding evaluation, and identification of different molecular processes (Aminpour et al., 2019; Geng et al., 2019; Hospital et al., 2015). The MD simulation process has a major role in the recognition of protein-ligand interactions and the protein conformational modifications at the atomic level. Hence, in the present study, the MD simulation method was used to evaluate the physicochemical characteristics of the four flavonoid inhibitors of tyrosinase, namely chrysin, quercetin, naringin, and kaempferol, in comparison with the enzyme-substrate complex.

\section{Methods}

\section{MD simulation}

The tyrosinase crystal structure (entry code: $2 \mathrm{Y} 9 \mathrm{X}$ ) (Bagherzadeh et al., 2015; Ismaya et al., 2011) was obtained from the PDB (http://www.rcsb.org). The characteristics of chrysin (CID: 5281607), kaempferol (CID: 5280863), quercetin (CID: 5280343), naringin (CID: 442428), and the substrate (L-DOPA, CID: 6047) were taken from PubChem (https://pubchem.ncbi.nlm.nih. 
gov/). The graphical AutoDock tool was applied to assess the permissible torsions of the ligand, characterize search space coordinates, and add polar hydrogen atoms to the protein (Morris et al., 1998). The docking process was then performed through a grid size of $20 \times 20 \times 14$ along the $\mathrm{X}, \mathrm{Y}$, and $\mathrm{Z}$ axes with $1 \AA$ spacing. The lowest binding energies of the enzyme-inhibitor and enzymesubstrate complexes were obtained using AutoDock Vina (Trott and Olson, 2010). To perform the MD simulation for the complexes, the GROMOS 53a6 (Gromacs 5.1 package) was applied (Van der Spoel et al., 2005). The enzyme-inhibitor and enzyme-substrate complexes with the lowest binding free energies were obtained by AutoDock Vina (Trott and Olson, 2010), and they were considered as the primary structures for the MD simulation process. The ProDrug program was used to provide the topological characteristics of chrysin, kaempferol, quercetin, naringin, and the substrate (Schüttelkopf and Van Aalten, 2004). In this study, complexes were solvated by the transferable intermolecular potential with 3 points (TIP3P) water model in a cubic box with a distance of $10 \AA$ from the furthest atom of the protein (Jorgensen et al., 1983). After solvation, $\mathrm{Na}^{+}$and $\mathrm{Cl}^{-}$ions were inserted to neutralize the system. Then, $\mathrm{NaCl}$ at the concentration of $150 \mathrm{mM}$ was introduced in the system (Batoulis et al., 2016; Reis et al., 2014), and energy minimization was performed using the steepest descent method. Each system was equilibrated by $1 \mathrm{~ns}$ MD simulation in the canonical (NVT) ensemble and $1 \mathrm{~ns}$ MD simulation in the isothermal-isobaric (NPT) ensemble using position restraints on the heavy atoms of the protein to allow for the equilibration of the solvent. The Nose-Hoover thermostat constant was used to fix the temperature of the system at $300 \mathrm{~K}$. To maintain the pressure of the system at a fixed 1 bar pressure, the Parrinello-Rahman pressure coupling method was used (Akya et al., 2019). The electrostatic interactions were calculated using the Particle Mesh Ewald (PME) method with 1.0 nm shortrange electrostatic and van der Waals cutoffs (Farasat et al., 2017; Ochoa et al., 2018). Consequently, the process of $50 \mathrm{~ns} \mathrm{MD}$ simulation for each complex of the enzyme-substrate/inhibitor was performed with time steps of $2 \mathrm{fs}$ on the equilibrated systems.

\section{PMF analysis of the enzyme-substrate and enzyme-inhibitor complexes}

Umbrella sampling (US) is a method applied to obtain the binding free energy pattern, which is often re- ferred to as PMF (Potential of Mean Force) along a special reaction coordinate including the protein-protein separation distance (You et al., 2019). The application of a physical reaction coordinate can give further structural insights (Naughton et al., 2018). In this study, the binding energies of the enzyme-substrate and enzyme-inhibitor complexes were calculated from PMF using the US method. In the first step, the MD simulation was performed to drive the substrate/inhibitor far away from the enzyme which was stable during the simulation process. In the next step, 50 configurations were created along the $z$-axis coordinate. The $z$ coordinates of the COM (center of mass) interval among the substrate/inhibitor and enzyme differed by $0.5 \AA$ in each configuration with the force constant of $10 \mathrm{kcal} / \mathrm{mol} / \AA)$. The equilibration process for each window was performed in a period of $10 \mathrm{~ns}$. Moreover, a $10 \mathrm{~ns}$ production run was continued for sampling (Gheibi et al., 2019; Lemkul and Bevan, 2010). Finally, the PMF pattern was provided by the Weighted Histogram Analysis (WHAM) method, which was carried out by GROMACS as "g_wham" command (Zeng et al., 2016). Moreover, for a better recognition of the MD process, the RMSF (root mean square fluctuation) (Chen et al., 2016; Mahapatra et al., 2018), RMSD (root mean square deviation) (Kaur et al., 2019; Shen et al., 2012), Rg (Radius of gyration) (Anantram et al., 2018; Farasat et al., 2017; Lobanov et al., 2008), the inhibitor and substrate distance from the amino acids of the enzyme active site (Corvo et al., 2013; Kato et al., 2017), and the van der Waals and the electrostatic energy of each system were analyzed using GROMACS tools in the period of simulation (Fried and Boxer, 2017; Schutt et al., 2015). Eventually, the final PDB file of MD simulation was plotted using Pymol software (Stourac et al., 2019). The LigPlot software (Laskowski and Swindells, 2011) was then used to analyze the H-bond and hydrophobic reactions of the complexes under investigation.

\section{Results and discussion}

\section{Molecular docking and the MD simulation analysis}

To observe the binding mode of flavonoid compounds on tyrosinase, the molecular docking process was performed. The docking results indicated that the substrates, chrysin, kaempferol, quercetin, and naringin bind to the enzyme active site with the lowest binding energies of $-6.1,-5.3,-5.9,-5.6$, and $-6.2 \mathrm{kcal} / \mathrm{mol}$, respecti- 
Table 1. Amino acids of tyrosinase involved in H-bond and hydrophobic interactions

\begin{tabular}{c|l|c|l}
\hline No. & \multicolumn{1}{|c|}{ Complexes } & $\begin{array}{c}\text { Amino acids } \\
\text { in H-bond } \\
\text { interaction }\end{array}$ & \multicolumn{1}{|c}{ Amino acids in hydrophobic interaction } \\
\hline 1 & tyrosinase-substrate & Met280 & $\begin{array}{l}\text { Val248, Asn260, His259, Phe264, Gly281, Val283, Ala286, His61, Ser282, } \\
\text { His263, His244 }\end{array}$ \\
\hline 2 & tyrosinase-chrysin & His85 & Glu322, Thr324, His244, Asn260, Val283, Asn81 \\
\hline 3 & tyrosinase-kaempferol & His85 & Thr324, Glu322, Asn81, Val283, His244, Val248, Asn260 \\
\hline 4 & tyrosinase-quercetin & His85 & Asn81, Val283, Asn260, Val248, Glu322, Thr324 \\
\hline 5 & tyrosinase-naringin & Val283 & Arg268, Gly281, Pro277, Phe264, Asn260, Pro284, Asn81, His85, Glu322, Thr324 \\
\hline
\end{tabular}

vely. It should be noted that the conformation of the enzyme-inhibitor and the enzyme-substrate complexes were applied as models for MD simulation based on the lowest binding free energy. The 2D and 3D images of the enzyme-substrate/inhibitor complexes after $50 \mathrm{~ns}$ of MD simulation are shown in Figure 1 and Table 1. As illustrated in 2D images, in the enzyme-substrate complex, the substrate (L-DOPA) had hydrophobic interactions with His61, His244, Val248, His259, Asn260, His263, Phe264, Gly281, Ser282, Val283, and Ala286 and a hydrogen interaction with Met280 residue (Fig. 1A). In the enzyme-inhibitor (chrysin) complex, chrysin had hydrophobic interactions with Asn81, His244, Asn260, Val283, Glu322, and Thr324 and a hydrogen interaction with His85 (Fig. 1B). Kaempferol had hydrophobic interactions with Asn81, His244, Val248, Asn260, Val283, Glu322, and Thr324 and a hydrogen interaction with His85 (Fig. 1C). Quercetin had hydrophobic interactions with Asn81, Val248, Asn260, Glu322, and Thr324 and a hydrogen interaction with His85 (Fig. 1D). Naringin had hydrophobic interactions with Asn81, His85, His244, Asn260, Phe264, Arg268, Pro277, Pro284, Glu322, and Thre324 and a hydrogen interaction with Val283 (Fig. 1E). His244, Glu256, Phe264, Val283, and Asn260 residues play a significant role in the active site of tyrosinase (Ismaya et al., 2011). As shown in Figure 1, all the above mentioned residues except Glu256 participated in the binding of the enzyme to the substrate or the inhibitors. Furthermore, the positions of the substrate and the inhibitors in relation to the above mentioned residues in the enzyme active site are shown in 3D images (Fig. 1).

RMSD is an essential parameter applied to predict the system equilibration during the simulation (Kaur et al. 2019; Shen et al. 2012). After the simulation pro- cess, to ensure that the system was equilibrated properly, the RMSD profiles of the enzyme-substrate/inhibitors complexes were analyzed during a $50 \mathrm{~ns}$ simulation process. The RMSD profiles of the aforementioned complexes are illustrated in Figure 2, which shows that the systems were equilibrated approximately after $5 \mathrm{~ns}$. The RMSD average values of the enzyme-substrate complex, enzyme-chrysin, enzyme-kaempferol, enzyme-quercetin, and enzyme-naringin in the last $5 \mathrm{~ns}$ of the simulation were $0.35,0.31,0.27,0.27$, and $0.27 \mathrm{~nm}$, respectively. It should be noted that all the complexes (substrateenzyme, chrysin-enzyme, quercetin-enzyme, kaempferolenzyme, and naringin-enzyme) were stabilized after $5 \mathrm{~ns}$. The RMSD differences between the substrate-enzyme and inhibitor-enzyme complexes were below $0.2 \mathrm{~nm}$, which indicates that the values are adequate (Chen et al., 2018).

To study the substrate/inhibitor effects on the system during the simulation process, other structural parameters, including gyration and RMSF, were also calculated.

The $\mathrm{Rg}$ (radius of gyration) demonstrates the protein contraction during the simulation (Anantram etal., 2018; Farasat et al., 2017; Lobanov et al., 2008). To evaluate the effects of inhibitors on enzyme contraction during the simulation, the gyration of each system was measured (Fig. 3). The Rg average values of the last $10 \mathrm{~ns}$ for the tyrosinase-substrate complex, tyrosinase-chrysin, tyrosinase-kaempferol, tyrosinase-quercetin, and tyrosinase-naringin were $2.09,2.07,2.07,2.07$, and $2.06 \mathrm{~nm}$, respectively. Our results showed no significant difference between the Rg values of the enzyme-substrate/inhibitor complexes. Thus, this study revealed no difference in tyrosinase enzyme contraction in the enzymesubstrate and enzyme-inhibitor complexes during the 
A
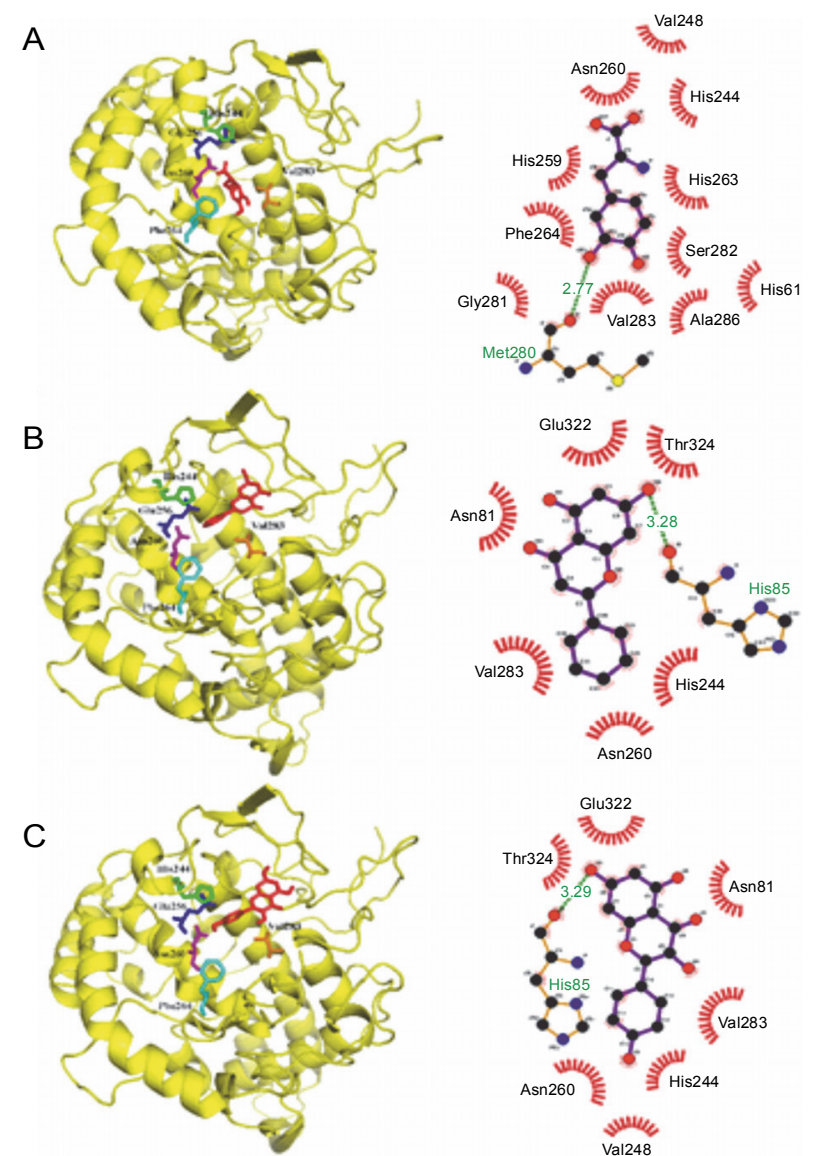

D

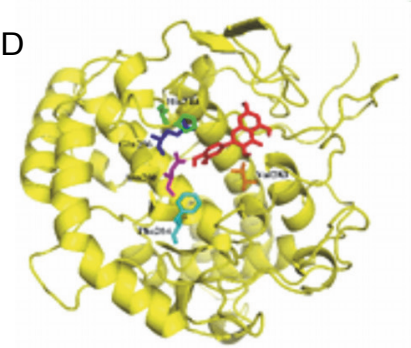

E

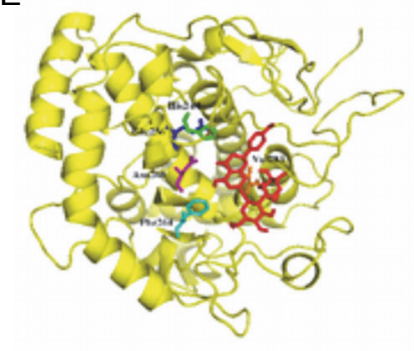

simulation. In 2018, Hassan M. et al. evaluated several anticarcinoma compounds by targeting SFRP4 through molecular modeling, docking, and dynamic simulation studies. The Rg time graph showed that residual backbone and the folding of the receptor protein were steadily stable after the binding of inhibitors, which confirmed our results (Hassan et al., 2018).

The RMSF value provides a better understanding of the protein flexibility and structural fluctuations (Chen et al., 2016; Mahapatra et al., 2018). To define the flexible regions of the enzyme in the enzyme-substrate/inhibitor complexes, the RMSF values of $\mathrm{C} \alpha$ atoms of the protein were evaluated. The RMSF values of the five complexes of the enzyme-substrate and enzyme-inhibitors are shown in Figure 4A. As shown in the figure, all the regions except for amino acid residues 203-221 had almost similar RMSF values. The fluctuation of the enzyme-substrate complex in the abovementioned residues differed from that in the enzyme-inhibitor complexes (Fig. 4 A/B). As demonstrated in Figure 4C, in Glu256 and Val283 residues, the fluctuations of the enzyme-inhibitor complex were smaller than those of the enzymesubstrate complex. These results confirmed that the inhibitors interact with the above residues, which leads to a decrease in fluctuation. This implies that in the presence of inhibitors (chrysin, naringin, quercetin, and kaempferol), the enzyme was less flexible in the vicinity of Glu256 and Val283 residues than in the presence of the substrate, which caused lesser flexibility and fluctuation of the enzyme. The RMSF values of the enzyme active site in the enzyme-substrate/inhibitor complexes are shown in Figure 4C. Similar findings of a lesser flexibility of protein when bound to various molecules demonstrated by molecular docking and molecular simulation approaches confirmed our observations (Gheibi et al., 2020).

\section{Distance monitoring of key amino acids of the enzyme active site from the substrate and inhibitors}

An approach to study the interaction of a substrate or

Fig. 1. The interactions of enzyme-substrate/inhibitor created by Lig-plot and Pymol software. A) the 3D and 2D images of tyrosinase-substrate complex, B) tyrosinase-chrysin, C) tyrosinase-kaempferol, D) tyrosinase-quercetin, and E) tyrosinasenaringin; the $3 \mathrm{D}$ images indicate that the substrate and the inhibitor bind to the active site of the enzyme; the 2D images demonstrate the amino acids involved in the substrate/inhibitor interactions an inhibitor with an enzyme is to evaluate the interval changes between the major amino acids of the active site and the defined compounds (Corvo et al., 2013; Kato et al., 2017). Thus, the distance between the key amino acids in the active site of the enzyme and the compounds was analyzed during the simulation. His244 is one of the major amino acids necessary for tyrosinase activity. Stu- 


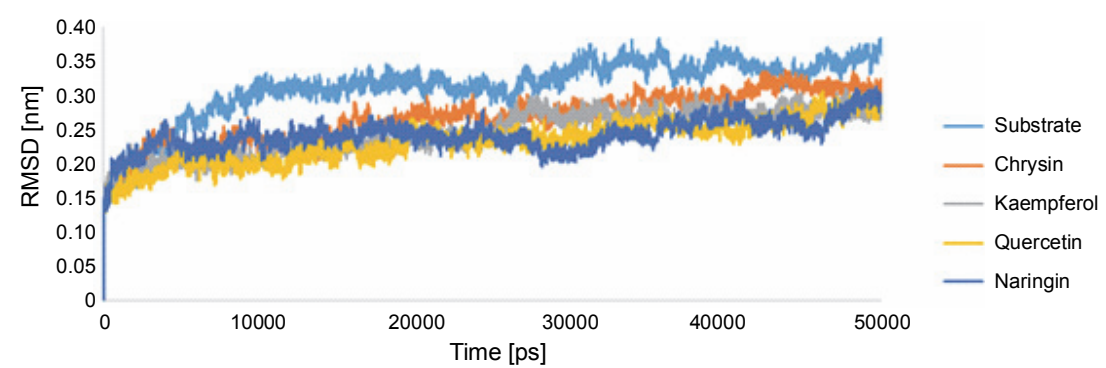

Fig. 2. The root mean square deviation (RMSD) values of $\mathrm{C} \alpha$ of the enzyme-substrate/inhibitor complexes

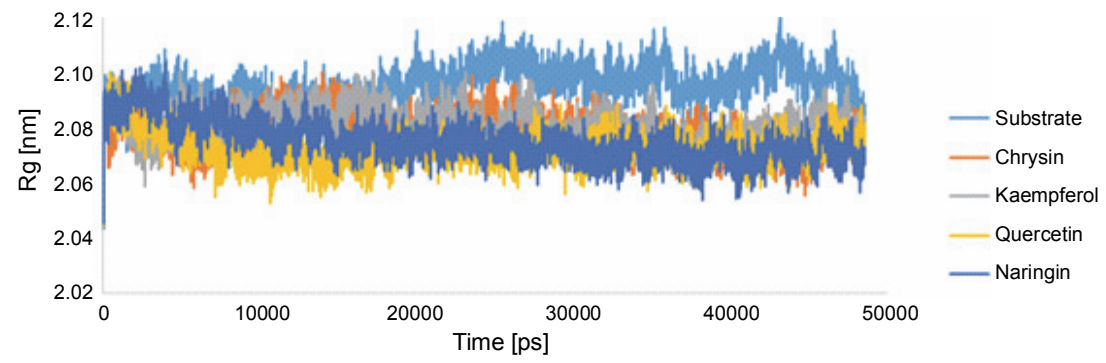

Fig. 3. The radius of gyration values of the enzyme-substrate/inhibitor complexes; the Rg average values of the enzyme-substrate complex, enzyme-chrysin, enzyme-kaempferol, enzyme-quercetin, and enzyme-naringin are shown
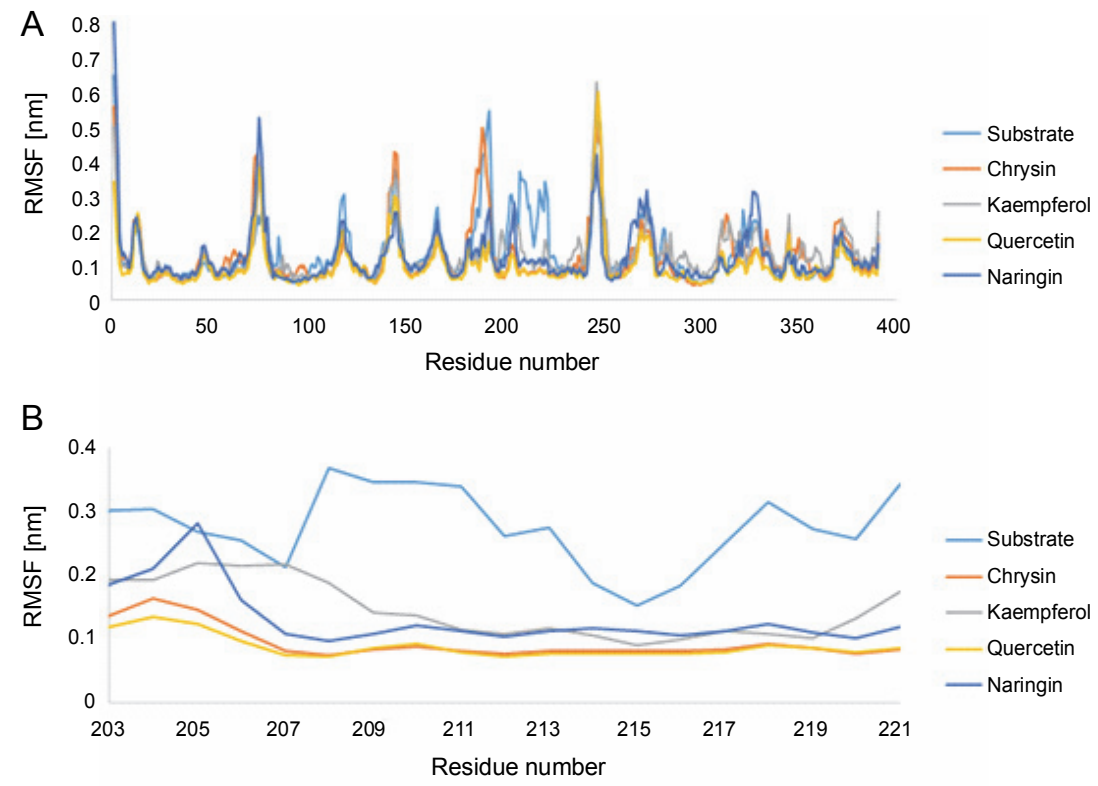

C

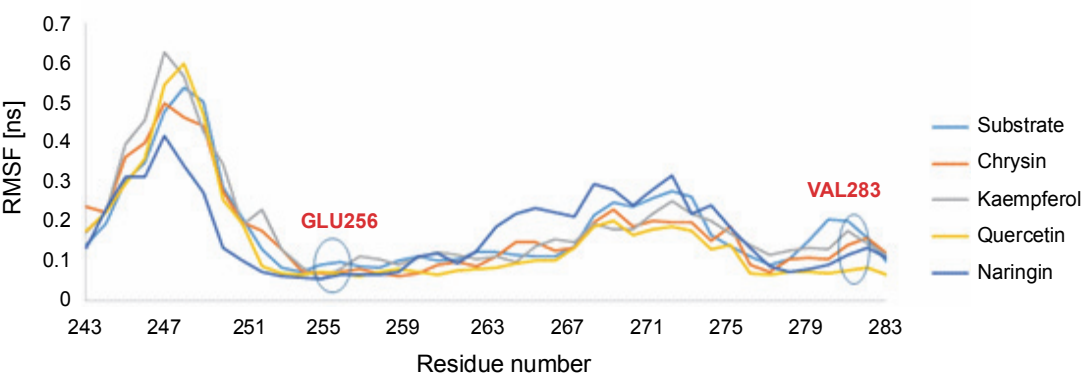

Fig. 4. The RMS fluctuation values of the enzyme-substrate/inhibitor complexes. A) the RMS fluctuations of the enzymesubstrate/inhibitor complexes for all tyrosinase amino acid residues; B) The RMSF values of the enzyme-substrate/inhibitor complexes for 203-221 residues; C) the RMSF values of the enzyme-substrate/inhibitor complexes in 243-283 residues 


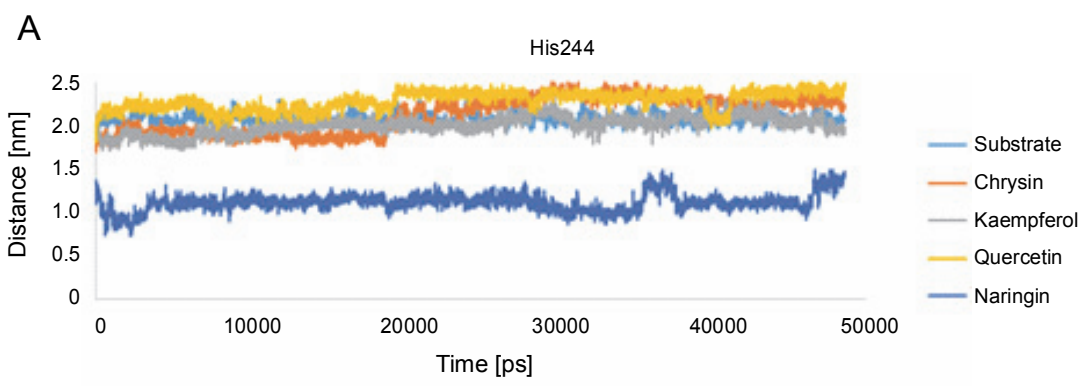

\section{B}

Glu256

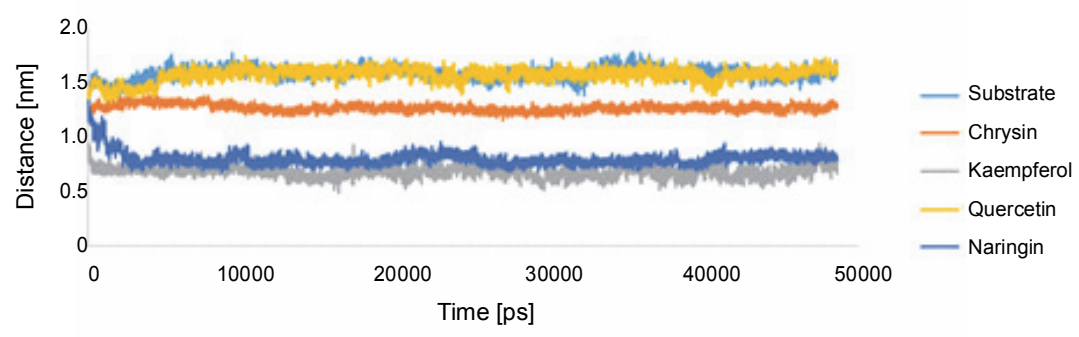

C
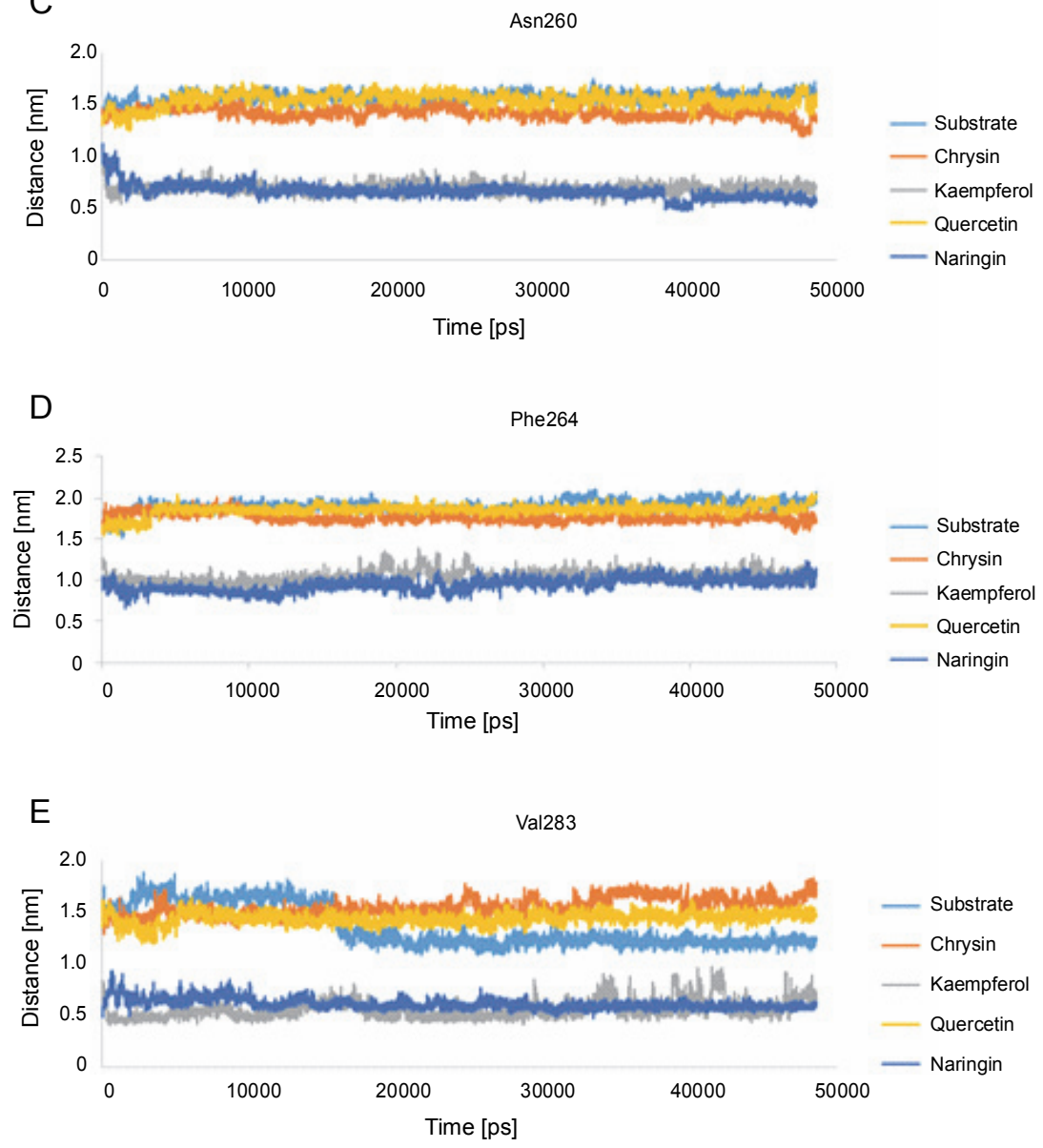

Fig. 5. The distance plot of the active sites of tyrosinase A) His244, B) Glu256, C) Asn260, D) Phe264, and E) Val283 residues and substrate/inhibitors 


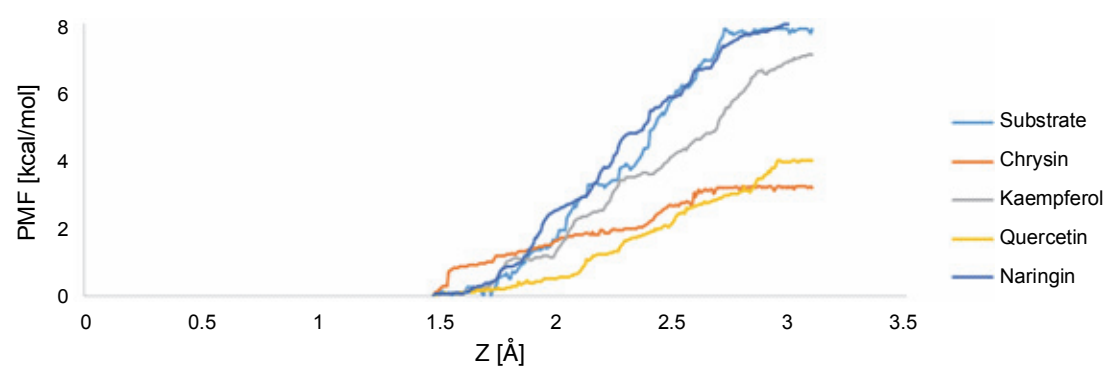

Fig. 6. The binding free energy profile of the enzyme dissociated from the enzyme-substrate/inhibitor complexes (enzyme-substrate, enzyme-chrysin, enzyme-kaempferol, enzyme-quercetin, and enzyme-naringin)

dying the interval changes of His244 and the evaluated compounds demonstrated that the substrate, chrysin, quercetin, and kaempferol possessed a similar interval average $(2 \mathrm{~nm})$, while this distance for naringin was nearly $1.2 \mathrm{~nm}$ (Fig. 5A). Hence, among the four inhibitors tested, naringin had a stronger interaction with tyrosinase; thus, it may also have a higher inhibitory effect than other inhibitors (chrysin, quercetin, and kaempferol).

Furthermore, the distance between Glu256, Asn260, Phe264, and Val283 residues of tyrosinase and the inhibitors was evaluated. The distances obtained for the complexes of Glu256, Asp260, Phe264, and Val283 of the enzyme with chrysin and quercetin were similar to those observed in the enzyme-substrate complex. For kaempferol and naringin inhibitors, the distance was shorter, and the inhibitors were closer than the substrate to the enzyme active site. The distance between Glu256 and the substrate and quercetin was almost similar $(1.5 \mathrm{~nm})$, while this distance was shorter (nearly $1.3 \mathrm{~nm}$ ) for chrysin and below $1 \mathrm{~nm}$ for naringin and kaempferol (Fig. 5B). The distance between Asn260 and the substrate, chrysin, and quercetin was nearly the same $(1.5 \mathrm{~nm})$, while the distance for kaempferol and naringin was approximately $1 \mathrm{~nm}$ (Fig. 5C). In evaluating the distance between Phe264 and the substrate/inhibitors, the results showed that the distance between Phe264 and the substrate, quercetin, and chrysin was $2 \mathrm{~nm}$ and was stable during the simulation. For naringin and kaempferol, the distance was $1 \mathrm{~nm}$ (Fig. 5D). Furthermore, the distances between Val283 and chrysin and between Val283 and quercetin were similar $(1.5 \mathrm{~nm})$ during the simulation. Moreover, the distance between Val283 and the substrate was unstable, and some fluctuations were observed (from 1.5 to $1.1 \mathrm{~nm}$ ). The distances between this residue and kaempferol or naringin were shorter, approximately $0.5 \mathrm{~nm}$ (Fig. $5 \mathrm{E}$ ). Because of the presence of phenolic compounds in the structure of inhibitors, the inhibitors strongly bound to the active site of tyrosinase (hydrophobic region), which leads to a better inhibitory effect (Panzella and Napolitano, 2019). These results show a stronger binding of the inhibitors to the enzyme than with the substrate. To verify these findings, the binding energies of the enzyme-substrate/inhibitor complexes were calculated using the US method. Several studies have proved that the interaction between the active site of the enzyme (amino acids of the active site) and inhibitors plays a major role in maintaining the stability of the enzyme-inhibitor complex. This reveals that when the distance between the inhibitor and the amino acids of the active site decreases, the inhibitory effects of inhibitors increase and the enzyme-inhibitor complex becomes more stable (Amin et al., 2013; Kato et al., 2017; Maria-Solano et al., 2018).

\section{Binding energy calculation}

The binding energy calculation showed the following binding energy values for the complex of substrate, chrysin, kaempferol, quercetin, and naringin with the enzyme: $-7.8,-3.1,-7.1,-3.9$, and $-8.4 \mathrm{kcal} / \mathrm{mol}$, respectively (Fig. 6). For these complexes (substrate-enzyme and inhibitor-enzyme), the results of the binding energy analysis indicated that among all inhibitors, naringin bound to the enzyme more strongly than the substrate. In recent studies, Farasat A (2017) and Gheibi N. et al. (2019b) demonstrated that when the binding energy decreases, the stability of the complex increases.

\section{Analysis of the electrostatic and van der Waals energies of substrate-enzyme and inhibitor-enzyme complexes}

To study the characteristics of noncovalent energies between tyrosinase-substrate and tyrosinase-inhibitors, 
A

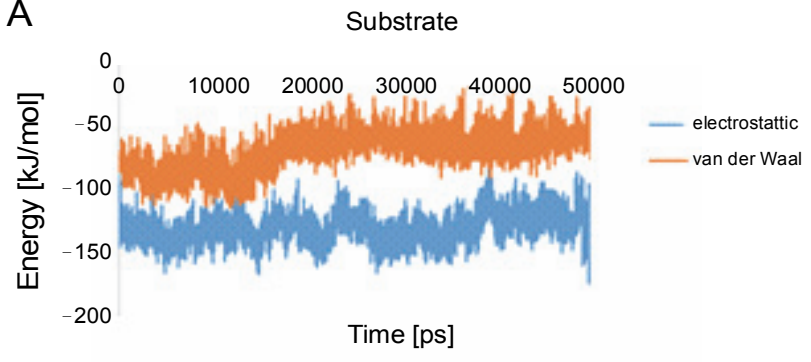

B

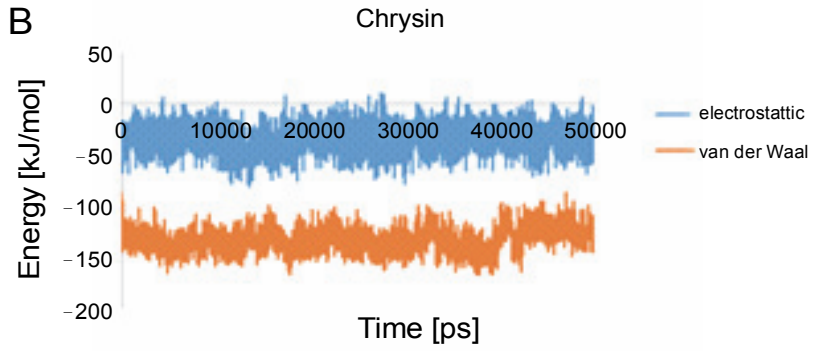

C
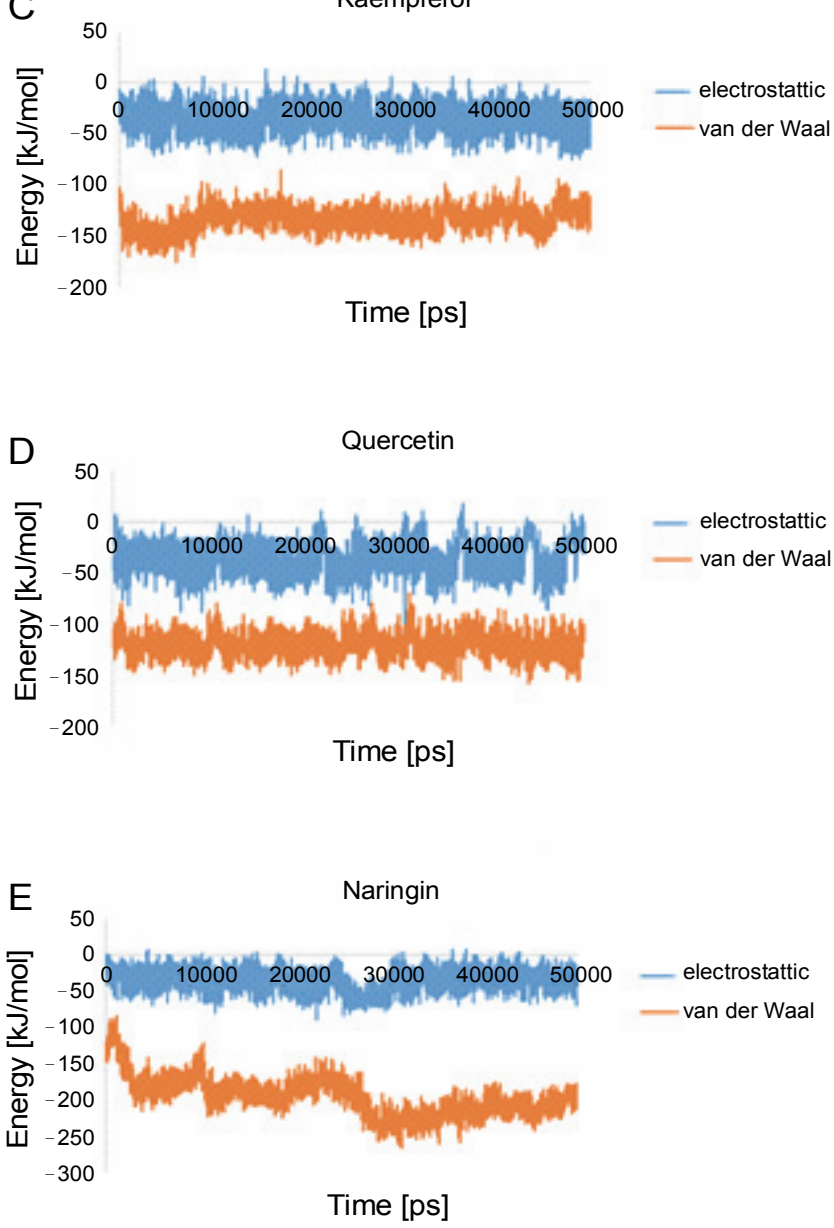

Fig. 7. The van der Waals and electrostatic energies between the enzyme and the substrate/inhibitor; the van der Waals and electrostatic energies of A) enzyme-substrate; B) enzyme-chrysin; C) enzyme-kaempferol; D) enzyme-quercetin; and $\mathrm{E}$ ) enzyme-naringin complexes the electrostatic and van der Waals interactions were evaluated during the simulation. The van der Waals and electrostatic energies play a major role in the binding of the substrate and the inhibitors to the active site of the enzyme (Fried and Boxer, 2017; Schutt et al., 2015). Our results revealed that in an enzyme-substrate complex, the electrostatic energy had a greater role than van der Waals energy. In the enzyme-substrate complex, the van der Waals and electrostatic energies were calculated as -65 and $-129 \mathrm{~kJ} / \mathrm{mol}$, respectively, for the last $10 \mathrm{~ns}$ of simulation. These findings verified that the electrostatic energy was almost two times greater than the van der Waals energy. Previous studies have confirmed that the electrostatic interactions play the main role in the binding of the substrate to the enzyme (Baudry et al., 2003; Ellis etal., 1995; Modi etal., 1996). On the other hand, the evaluation of the electrostatic and van der Waals interactions in the enzyme-inhibitor complexes indicated that the van der Waals interactions were 3-4 times higher than the electrostatic interactions (Fig. 7). These results showed that the van der Waals forces play the main role in the enzyme-inhibitor complexes. Bonnet and Bryce (2004) showed that the van der Waals interactions play the major role in the binding of the enzyme to the inhibitor, which confirms our results. Additionally, because of the hydrophobic content, the flavonoid inhibitors tend to interact with enzymes through hydrophobic and van der Waals interactions (Li et al., 2009). The enzyme-naringin complex possessed the highest van der Waals energy, which was 4 times higher than the electrostatic energy. In enzyme-chrysin, enzyme-kaempferol, and enzyme-quercetin complexes, the van der Waals and electrostatic energies were -150 and $-50 \mathrm{~kJ} / \mathrm{mol}$, respectively (Fig. 7).

According to previous studies, the inhibition constants (Ki) of naringin, quercetin, and chrysin were estimated to be $3.04,7.44$, and $7.90 \mathrm{mM}$, respectively (Gheibi et al., 2016; Taherkhani and Gheibi, 2014). These findings confirm our observations that naringin would be the best flavonoid inhibitor of the tyrosinase enzyme (Gheibi et al., 2016). Because of broad utilization and high therapeutic potential of naringin as an inhibitor in biological and clinical fields, it is suggested that naringin can be tested and possibly used as a therapeutic agent in various human disorders (Bharti et al., 2014). In another study (Taherkhani and Gheibi, 2014), kaempferol and quercetin were used as tyrosinase inhibitors, and it was found that kaempferol exerts a stronger 
inhibitory effect on tyrosinase than quercetin, which was also confirmed by our results.

\section{Conclusion}

Our findings revealed that among the four flavonoid inhibitors studied, namely chrysin, kaempferol, naringin, and quercetin, naringin exerts the best inhibitory effects on tyrosinase, which makes it an appropriate candidate as a whitening agent in skin disorders. In fact, the flavonoid derivatives restricted the enzyme activity. Nevertheless, compared to the other three flavonoid compounds, naringin might particularly be considered as a potential therapeutic inhibitor for the treatment and prevention of melanoma and skin hyperpigmentation disorders, which are caused by overactivity of tyrosinase.

\section{Acknowledgment}

The authors are grateful to the Research Council of Qazvin University of Medical Sciences for the support received.

\section{References}

Abdelrasool M., Said A. (2017) Literature review on tyrosinase enzyme.

Akya A., Farasat A., Ghadiri K., Rostamian M. (2019) Identification of hla-i restricted epitopes in six vaccine candidates of leishmania tropica using immunoinformatics and molecular dynamics simulation approaches. Infect. Genet. Evol. 75: 103953.

Amin S.R., Erdin S., Ward R.M., Lua R.C., Lichtarge O. (2013) Prediction and experimental validation of enzyme substrate specificity in protein structures. Proc. Nat. Acad. Sci. 110(45): E4195-E4202.

Aminpour M., Montemagno C., Tuszynski J.A. (2019) An overview of molecular modeling for drug discovery with specific illustrative examples of applications. Molecules 24(9): 1693.

Anantram A., Kundaikar H., Degani M., Prabhu A. (2019) Molecular dynamic simulations on an inhibitor of anti-apoptotic Bcl-2 proteins for insights into its interaction mechanism for anti-cancer activity. J. Biomol. Struct. Dyn. 37(12): 3109-3121.

Bagherzadeh K., Shirgahi Talari F., Sharifi A., Ganjali M.R., Saboury A.A., Amanlou M. (2015) A new insight into mushroom tyrosinase inhibitors: docking, pharmacophore-based virtual screening, and molecular modeling studies. J. Biomol. Struct. Dyn. 33(3): 487-501.

Batoulis H., Schmidt T.H., Weber P., Schloetel J.-G., Kandt C., Lang T. (2016) Concentration dependent ion-protein interaction patterns underlying protein oligomerization behaviours. Sci. Rep. 6: 24131.

Baudry J., Li W., Pan L., Berenbaum M.R., Schuler M.A. (2003) Molecular docking of substrates and inhibitors in the catalytic site of cyp6b1, an insect cytochrome p450 monooxygenase. Protein Eng. 16(8): 577-587.

Bharti S., Rani N., Krishnamurthy B., Arya D.S. (2014) Preclinical evidence for the pharmacological actions of naringin: a review. Planta Med. 80(06): 437-451.

Chang T.-S. (2009) An updated review of tyrosinase inhibitors. Inter. J. Mol. Sci. 10(6): 2440-2475.

Chen H., Fu W., Wang Z., Wang X., Lei T., Zhu F., Li D., Chang S., Xu L., Hou T. (2018) Reliability of docking-based virtual screening for GPCR ligands with homology modeled structures: a case study of the angiotensin II type I receptor. ACS Chem. Neurosci. 10(1): 677-689.

Chen J., Wang J., Zhu W. (2016) Molecular mechanism and energy basis of conformational diversity of antibody spe 7 revealed by molecular dynamics simulation and principal component analysis. Sci. Rep. 6: 36900.

Choi J., Lee Y.-M., Jee J.-G. (2017) Thiopurine drugs repositioned as tyrosinase inhibitors. Inter. J. Mol. Sci. 19(1): 77.

Choi M.-H., Shin H.-J. (2016) Anti-melanogenesis effect of quercetin. Cosmetics 3(2): 18.

Corvo I., O’Donoghue A.J., Pastro L., Pi-Denis N., Eroy-Reveles A., Roche L., McKerrow J.H., Dalton J.P., Craik C.S., Caffrey C.R. (2013) Dissecting the active site of the collagenolytic cathepsin 13 protease of the invasive stage of Fasciola hepatica. PLoS Neglected Tropical Dis. 7(7): e2269.

Da Hae G., Jo J.M., Kim S.Y., Kim J.W. (2019) Tyrosinase inhibitors from natural source as skin-whitening agents and the application of edible insects: a mini review. Inter. J. Clin. Nutr. Diet. 5: 141.

da Silva A.P., Silva N.F., Andrade E.H.A., Gratieri T., Setzer W.N., Maia J.G.S., da Silva J.K.R. (2017) Tyrosinase inhibitory activity, molecular docking studies and antioxidant potential of chemotypes of lippia origanoides (verbenaceae) essential oils. PloS One. 12(5). https://doi.org/ 10.1371/journal.pone. 0175598

Deri B., Kanteev M., Goldfeder M., Lecina D., Guallar V., Adir N., Fishman A. (2016) The unravelling of the complex pattern of tyrosinase inhibition. Sci. Rep. 6: 34993.

Ellis S.W., Hayhurst G.P., Smith G., Lightfoot T., Wong M.M., Simula A.P., Ackland M.J., Sternberg M.J., Lennard M.S., Tucker G.T. (1995) Evidence that aspartic acid 301 is a critical substrate-contact residue in the active site of cytochrome p450 2d6. J. Biol. Chem. 270(49): 29055-29058.

Farasat A., Rahbarizadeh F., Hosseinzadeh G., Sajjadi S., Kamali M., Keihan A.H. (2017) Affinity enhancement of nanobody binding to egfr: In silico site-directed mutagenesis and molecular dynamics simulation approaches. J. Biomol. Struct. Dyn. 35(8): 1710-1728.

Fried S.D., Boxer S.G. (2017) Electric fields and enzyme catalysis. Ann. Rev. Biochem. 86: 387-415.

Geng H., Chen F., Ye J., Jiang F. (2019) Applications of moleculardynamics simulation in structure prediction of peptides and proteins. Comput. Struct. Biotechnol. J. 17: 1162-1170.

Gheibi N., Ghorbani M., Shariatifar H., Farasat A. (2019) In silico assessment of human calprotectin subunits (s100a8/a9) in presence of sodium and calcium ions using molecular dynamics simulation approach. PloS One 14(10): e0224095. 
Gheibi N., Ghorbani M., Shariatifar H., Farasat A. (2020) Effects of unsaturated fatty acids (arachidonic/oleic acids) on stability and structural properties of calprotectin using molecular docking and molecular dynamics simulation approach. PloS One 15(3): e0230780.

Gheibi N., Zavareh S.H., Behbahani G.R., Haghbeen K., SiratiSabet M., Ilghari D., Chegini K.G. (2016) Comprehensive kinetic and structural studies of different flavonoids inhibiting diphenolase activity of mushroom tyrosinase. Appl. Biochem. Microbiol. 52(3): 304-310.

Harris Z., Donovan M.G., Branco G.M., Limesand K.H., Burd R. (2016) Quercetin as an emerging anti-melanoma agent: A four-focus area therapeutic development strategy. Front. Nutr. 3: 48.

Hassan M., Azhar M., Abbas Q., Raza H., Moustafa A.A., Shahzadi S., Ashraf Z., Seo S. (2018) Finding novel anti-carcinomas compounds by targeting sfrp4 through molecular modeling, docking and dynamic simulation studies. Curr. Comput. Aided Drug Design. 14(2): 160-173.

Hassani S., Gharechaei B., Nikfard S., Fazli M., Gheibi N., Hardré R., Legge R.L., Haghbeen K. (2018) New insight into the allosteric effect of 1-tyrosine on mushroom tyrosinase during l-dopa production. Inter. J. Biol. Macromol. 114: 821-829.

Horáková L'. (2011) Flavonoids in prevention of diseases with respect to modulation of ca-pump function. Interdiscip. Toxicol. 4(3): 114-124.

Hospital A., Goñi J.R., Orozco M., Gelpí J.L. (2015) Molecular dynamics simulations: advances and applications. Adv. Appl. Bioinform. Chem. AABC. 8: 37.

Ismaya W.T., Rozeboom H.J., Weijn A., Mes J.J., Fusetti F., Wichers H.J., Dijkstra B.W. (2011) Crystal structure of agaricus bisporus mushroom tyrosinase: identity of the tetramer subunits and interaction with tropolone. Biochemistry 50(24): 5477-5486.

Jorgensen W.L., Chandrasekhar J., Madura J.D., Impey R.W., Klein M.L. (1983) Comparison of simple potential functions for simulating liquid water. J. Chem. Phys. 79(2): 926-935.

Kato A., Nakagome I., Nakagawa S., Kinami K., Adachi I., Jenkinson S.F., Désiré J., Blériot Y., Nash R.J., Fleet G.W. (2017) In silico analyses of essential interactions of iminosugars with the hex a active site and evaluation of their pharmacological chaperone effects for tay-sachs disease. Organic Biomol. Chem. 15(44): 9297-9304.

Kaur G., Pandey B., Kumar A., Garewal N., Grover A., Kaur J. (2019) Drug targeted virtual screening and molecular dynamics of lipuprotein of mycobacterium tuberculosis and mycobacterium leprae. J. Biomol. Struct. Dyn. 37(5): 1254-1269.

Laskowski R.A, Swindells M.B. (2011) Ligplot+: multiple ligand-protein interaction diagrams for drug discovery. ACS Publications.

Lemkul J.A., Bevan D.R. (2010) Assessing the stability of alzheimer's amyloid protofibrils using molecular dynamics. J. Phys. Chem. B 114(4): 1652-1660.

Li Y.Q., Zhou F.C., Gao F., Bian J.S., Shan F. (2009) Comparative evaluation of quercetin, isoquercetin and rutin as inhibitors of $\alpha$-glucosidase. J. Agric. Food Chem. 57(24): 11463-11468.

Lin Y.-S., Chen H.-J., Huang J.-P., Lee P.-C., Tsai C.-R., Hsu T.-F., Huang W.-Y. (2017) Kinetics of tyrosinase inhibitory activity using Vitis vinifera leaf extracts. BioMed Res. Int. 2017: 5232680 .

Liu Smith F., Meyskens F.L. (2016) Molecular mechanisms of flavonoids in melanin synthesis and the potential for the prevention and treatment of melanoma. Mol. Nutr. Food Res. 60(6): 1264-1274.

Lobanov M.Y., Bogatyreva N., Galzitskaya O. (2008) Radius of gyration as an indicator of protein structure compactness. Mol. Biol. 42(4): 623-628.

Mahapatra M.K., Bera K., Singh D.V., Kumar R., Kumar M. (2018) In silico modelling and molecular dynamics simulation studies of thiazolidine based PTP1b inhibitors. J. Biomol. Struct. Dyn. 36(5): 1195-1211.

Maria-Solano M.A., Serrano-Hervás E., Romero-Rivera A., Iglesias-Fernández J., Osuna S. (2018) Role of conformational dynamics in the evolution of novel enzyme function. Chem. Commun. 54(50): 6622-6634.

Modi S., Paine M., Sutcliffe M., Lian L.-Y., Primrose W., Wolf C., Roberts G. (1996) A model for human cytochrome p450 $2 d 6$ based on homology modeling and nmr studies of substrate binding. Biochemistry 35(14): 4540-4550.

Morissette M., Litim N., Di Paolo T. (2018) Natural phytoestrogens: A class of promising neuroprotective agents for parkinson disease. Discovery and development of neuroprotective agents from natural products. Elsevier: 9-61.

Morris G.M., Goodsell D.S., Halliday R.S., Huey R., Hart W.E., Belew R.K., Olson A.J. (1998) Automated docking using a lamarckian genetic algorithm and an empirical binding free energy function. J. Comput. Chem. 19(14): 1639-1662.

Naughton F.B., Kalli A.C., Sansom M.S. (2018) Modes of interaction of pleckstrin homology domains with membranes: toward a computational biochemistry of membrane recognition. J. Mol. Biol. 430(3): 372-388.

Ochoa R., Soler M.A., Laio A., Cossio P. (2018) Assessing the capability of in silico mutation protocols for predicting the finite temperature conformation of amino acids. Phys. Chem. Chem. Phys. 20(40): 25901-25909.

Panzella L., Napolitano A. (2019) Natural and bioinspired phenolic compounds as tyrosinase inhibitors for the treatment of skin hyperpigmentation: recent advances. Cosmetics 6(4): 57.

Popoola O.K., Marnewick J.L., Rautenbach F., Ameer F., Iwuoha E.I., Hussein A.A. (2015) Inhibition of oxidative stress and skin aging-related enzymes by prenylated chalcones and other flavonoids from helichrysum teretifolium. Molecules 20(4): 7143-7155.

Promden W., Viriyabancha W., Monthakantirat O., Umehara K., Noguchi H., De-Eknamkul W. (2018) Correlation between the potency of flavonoids on mushroom tyrosinase inhibitory activity and melanin synthesis in melanocytes. Molecules 23(6): 1403. 
Qiu T., Wu D., Yang L., Ye H., Wang Q., Cao Z., Tang K. (2018) Exploring the mechanism of flavonoids through systematic bioinformatics analysis. Front. Pharmacol. 9: 918.

Reis R.A.G., Bortot L.O., Caliri A. (2014) In silico assessment of s100a12 monomer and dimer structural dynamics: Implications for the understanding of its metal-induced conformational changes. JBIC J. Biol. Inorg. Chem. 19(7): $1113-1120$

SchuttT.C., Bharadwaj V.S., Granum D.M., Maupin C.M. (2015) The impact of active site protonation on substrate ring conformation in Melanocarpus albomyces cellobiohydrolase cel7b. Phys. Chem. Chem. Phys. 17(26): 16947-16958.

Schüttelkopf A.W., Van Aalten D.M. (2004) Prodrg: A tool for high-throughput crystallography of protein-ligand complexes. Acta Cryst. Sect. D: Biol. Cryst. 60(8): 1355-1363.

Shen M., Guan J., Xu L., Yu Y., He J., Jones G.W., Song Y. (2012) Steered molecular dynamics simulations on the binding of the appendant structure and helix $\beta 2$ in domainswapped human cystatin $C$ dimer. J. Biomol. Struct. Dyn. 30(6): 652-661.

Solimine J., Garo E., Wedler J., Rusanov K., Fertig O., Hamburger M., Atanassov I., Butterweck V. (2016) Tyrosinase inhibitory constituents from a polyphenol enriched fraction of rose oil distillation wastewater. Fitoterapia 108: 13-19.

Stourac J., Vavra O., Kokkonen P., Filipovic J., Pinto G., Brezovsky J., Damborsky J., Bednar D. (2019) Caver web 1.0: Identification of tunnels and channels in proteins and analysis of ligand transport. Nucl. Acids Res. 47(W1): W414-W422.

Sugumaran M., Barek H. (2016) Critical analysis of the melanogenic pathway in insects and higher animals. Int. J. Mol. Sci. 17(10): 1753.
Taherkhani N., Gheibi N. (2014) Inhibitory effects of quercetin and kaempferol as two propolis derived flavonoids on tyrosinase enzyme. Biotech. Health Sci. 1(2): e22242.

Trott O., Olson A.J. (2010) Autodock vina: Improving the speed and accuracy of docking with a new scoring function, efficient optimization, and multithreading. J. Comput. Chem. 31(2): 455-461.

Ullah S., Son S., Yun H.Y., Kim D.H., Chun P., Moon H.R. (2016) Tyrosinase inhibitors: a patent review (2011-2015). Expert Opin. Therap. Patents 26(3): 347-362.

Van der Spoel D., Lindahl E., Hess B., Groenhof G., Mark A.E., BerendsenH.J.C. (2005) GROMACS: fast, flexible, and free. J. Comput. Chem. 26(16): 1701-1718.

Yin S.-J., Si Y.X., Wang Z.-J., Wang S.-F., Oh S., Lee S., Sim S.M., Yang J.-M., Qian G.-Y., Lee J. (2011) The effect of thiobarbituric acid on tyrosinase: inhibition kinetics and computational simulation. J. Biomol. Struct. Dyn. 29(3): 463-470.

You W., Tang Z., Chang C.-A. (2019) Potential mean force from umbrella sampling simulations: What can we learn and what is missed? J. Chem. Theory Comput. 15(4): 2433-2443.

Zeng S., Zhou G., Guo J., Zhou F., Chen J. (2016) Molecular simulations of conformation change and aggregation of HIV-1 VPR13-33 on graphene oxide. Sci. Rep. 6: 24906.

Zhu L., Lu Y., Yu W.-G., Zhao X., Lu Y.-H. (2016) Anti-photoageing and anti-melanogenesis activities of chrysin. Pharm. Biol. 54(11): 2692-2700.

Zolghadri S., Bahrami A., Hassan Khan M.T., Munoz-Munoz J., Garcia-Molina F., Garcia-Canovas F., Saboury A.A. (2019) A comprehensive review on tyrosinase inhibitors. J. Enzyme Inhibition Med. Chem. 34(1): 279-309. 